\title{
Collectively enhanced chiral photon emission from an atomic array near a nanofiber
}

\author{
Ryan Jones, ${ }^{1}$ Giuseppe Buonaiuto, ${ }^{1,2}$ Ben Lang, ${ }^{1}$ Igor Lesanovsky, ${ }^{1,2}$ and Beatriz Olmos ${ }^{1,2}$ \\ ${ }^{1}$ School of Physics and Astronomy and Centre for the Mathematics \\ and Theoretical Physics of Quantum Non-Equilibrium Systems, \\ The University of Nottingham, Nottingham, NG7 2RD, United Kingdom \\ ${ }^{2}$ Institut für Theoretische Physik, Universität Tübingen, \\ Auf der Morgenstelle 14, 72076 Tübingen, Germany
}

(Dated: January 8, 2020)

\begin{abstract}
Emitter ensembles interact collectively with the radiation field. In the case of a one-dimensional array of atoms near a nanofiber, this collective light-matter interaction does not only lead to an increased photon coupling to the guided modes within the fiber, but also to a drastic enhancement of the chirality in the photon emission. We show that near-perfect chirality can be achieved already for moderately-sized ensembles, containing 10 to 15 atoms, by phase-matching a superradiant collective guided emission mode via an external laser field. This is of importance for developing an efficient interface between atoms and waveguide structures with unidirectional coupling, with applications in quantum computing and communication such as the development of non-reciprocal photon devices or quantum information transfer channels.
\end{abstract}

Introduction. The radiative properties of a group of emitters are determined by the electromagnetic field mode structure of their enviroment [1-9]. They can be modified, for example, by the presence of nearby metallic or dielectric surfaces and nanospheres, metamaterials or plasmonic nanowires, among others [10-17]. This phenomenon, first described by Purcell in the 1940s [18], has been studied extensively in a variety of contexts, and most prominently in systems involving quantum optical devices [19-22].

Structured environments such as photonic crystals and optical fibers support a finite number of guided electromagnetic field modes. These are particularly interesting as they can possess a significant longitudinal field component [23-27]. The field around the fiber has, thus, elliptical polarization, whose sign depends on the direction of propagation of the mode being forwards or backwards along the fiber. If the polarization of the dipole moment of a nearby emitter is aligned with that of a guided mode, the emission will occur predominantly into this mode, breaking the forwards-backwards propagation symmetry. This so-called chiral coupling [28] has been observed experimentally for circularly polarized atoms near an optical fiber of sub-wavelength thickness (nanofiber) [29-32], as well as for a variety of other emitter types coupled with guided structures [33, 34].

While it is well understood how the radiative properties of a single atom are altered by the presence of a nanofiber [35-38], much less is known about the behavior of atomic ensembles [39-52]. However, understanding this situation is of increasing importance, as collections of emitters near a nanofiber promise applications in quantum information routing and processing, e.g. as optical isolators and circulators where the light is emitted unidirectionally, or as generators of entangled atomic and photonic states [53-62]. These applications can only be realised experimentally by dramatically enhancing the

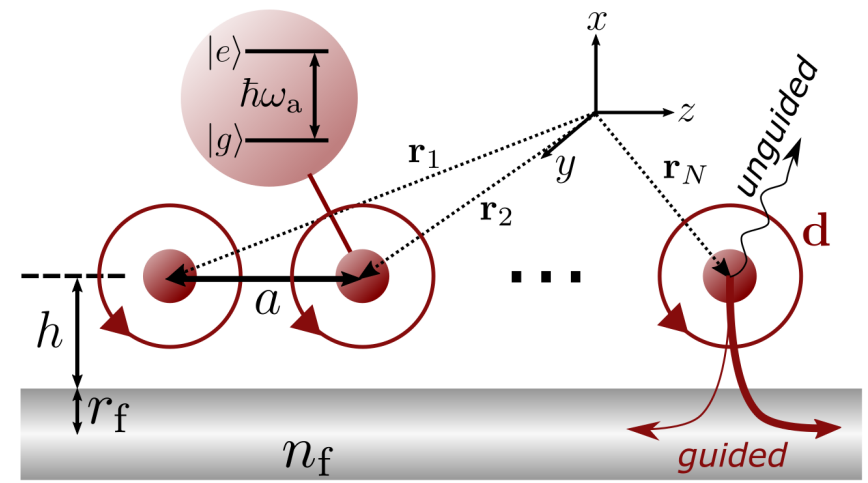

FIG. 1. System. Chain of $N$ identical two-level atoms (transition energy $\hbar \omega_{\mathrm{a}}$ ) with nearest neighbors separation $a$, placed at a distance $h$ above a nanofiber, which has radius $r_{\mathrm{f}}$ and refractive index $n_{\mathrm{f}}$. The dipole moment of the transition $|g\rangle \rightarrow|e\rangle$ in each atom is $\mathbf{d}=(i, 0,-1) d / \sqrt{2}$. The atoms are coupled both to the free field (unguided) and the nanofiber (guided) modes.

efficiency of the coupling between the emitters and the nanofiber. Moreover, the collective dissipative dynamics resulting from a competition between the coupling to the unguided modes of the free space and the guided ones of the nanofiber may result in the formation of complex many-body phases and phase transitions [21,61].

In this paper, we explore the question of whether chirality can be enhanced due to collective effects. To this end, we investigate the photon emission from a weakly driven one-dimensional array of atoms in the vicinity of a nanofiber. We show that, even for moderate number of atoms, the majority of photons is emitted into the fiber with near perfect chirality. This enhanced coupling is mediated by the appearance of a collective superradiant mode, which forms due to the presence of the nanofiber. When the laser driving field matches the phase profile of this mode, a dramatic increase in the efficiency 
of the atom-fiber coupling and at the same time complete unidirectionality of the photon emission is achieved. These results are of immediate relevance to current experimental efforts aiming to control light-matter interactions through the use of nanophotonic structures.

System and master equation. We consider a chain of $N$ identical atoms aligned parallel to the $z$-axis, with lattice constant $a$ (see Fig. 1). The internal structure of each atom is modelled as a two-level system, with ground $|g\rangle$ and excited $|e\rangle$ states separated by an energy $\hbar \omega_{\mathrm{a}}=\hbar 2 \pi c / \lambda_{\mathrm{a}}$, where $\lambda_{\mathrm{a}}$ is the wavelength of the $|g\rangle \rightarrow|e\rangle$ transition, whose dipole moments $\mathbf{d}$ are considered identical for all atoms. The atoms are placed at a distance $h$ above the surface of a cylindrical nanofiber with radius $r_{\mathrm{f}}$ and refractive index $n_{\mathrm{f}}>1$. In cylindrical coordinates $(r, \phi, z)$ the position of the atoms is given by $\mathbf{r}_{j}=\left(r_{\mathrm{f}}+h, 0,(j-1) a\right)$ for $j=1,2, \ldots, N$.

It can be shown that a nanofiber supports a small number of guided modes. We focus in the regime where the radius of the nanofiber obeys the so-called singlemode condition [63] $r_{\mathrm{f}}<2.405 \lambda_{\mathrm{a}} /\left(2 \pi \sqrt{n_{\mathrm{f}}^{2}-1}\right)$, such that the only modes supported by the nanofiber are the hybrid fundamental $\mathrm{HE}_{11}$. Throughout, we assume the nanofiber is made from silica, and we calculate the refractive index $n_{\mathrm{f}}$ as a function of the atomic transition wavelength $\lambda_{\mathrm{a}}$ using the Sellmeier equation [64].

Under the Born and Markov approximations, valid for the system sizes that we consider here, a quantum master equation, $\dot{\rho}=-\frac{i}{\hbar}[\mathcal{H}, \rho]+\mathcal{D}(\rho)$, describes the dynamics of the atoms through the reduced density matrix $\rho$ (see, e.g., [46]). The first term on the right hand side of this equation describes coherent dipole-dipole interactions among the atoms due to the exchange of virtual photons. The Hamiltonian that determines this coherent dynamics is

$$
\mathcal{H}=\hbar \sum_{i \neq j=1}^{N} V_{i j} \sigma_{i}^{\dagger} \sigma_{j}
$$

where $\sigma_{i}=|g\rangle_{i}\langle e|$. The rate of the dipole-dipole exchange between a pair of atoms $i$ and $j$ is characterised by the coefficient $V_{i j}$. The second term of the master equation encapsulates the action of dissipation,

$$
\mathcal{D}(\rho)=\sum_{i j=1}^{N} \Gamma_{i j}\left(\sigma_{j} \rho \sigma_{i}^{\dagger}-\frac{1}{2}\left\{\sigma_{i}^{\dagger} \sigma_{j}, \rho\right\}\right) .
$$

For a non-interacting chain of atoms, $\Gamma_{i j}=0$ for $i \neq j$ such that each atom decays independently with rate $\Gamma_{i i}$ which, due to the presence of the nanofiber, can vary significantly from the decay rate in vacuum, $\gamma$. However, in general, $\Gamma_{i j} \neq 0$ for $i \neq j$ (e.g. in a dense atomic chain in free space $[6,7]$ or near a nanofiber $[21,46]$ ), and the emission of photons from the chain becomes a collective process. This can be better understood by diagonalizing the coefficient matrix $\Gamma_{i j}=\sum_{c} M_{i c}^{\dagger} \gamma_{c} M_{c j}$. The dissipa- tor (2) then assumes the diagonal form

$$
\mathcal{D}(\rho)=\sum_{c=1}^{N} \gamma_{c}\left(J_{c} \rho J_{c}^{\dagger}-\frac{1}{2}\left\{J_{c}^{\dagger} J_{c}, \rho\right\}\right) .
$$

Here, it is apparent that the emission occurs via the collective jump operators $J_{c}=\sum_{j} M_{c j} \sigma_{j}$, which in general consist of superpositions of all single-atom lowering operators. The collective decay rates $\gamma_{c}$ (the eigenvalues of the matrix of $\Gamma_{i j}$ coefficients) can be much larger or smaller than $\Gamma_{i i}$, which is commonly referred to as superradiant and subradiant decay, respectively [1]. The exact form of $V_{i j}$ and $\Gamma_{i j}$, given in the Supplemental Material [65], depend strongly on the system parameters, such as $a, \lambda_{\mathrm{a}}, r_{\mathrm{f}}$ and $h$.

Collective decay modes. In order to gain an understanding of the collective decay modes, we first consider the (free space) situation where the fiber is absent (Fig. $2 \mathrm{a})$. For small interatomic separation, $a / \lambda_{\mathrm{a}} \ll 1$, the off-diagonal elements of $\Gamma_{i j}$ become comparable to the diagonal ones and superradiant $\left(\gamma_{c} \gg \gamma\right)$ and subradiant $\left(\gamma_{c} \ll \gamma\right)$ modes emerge. As the distance between the atoms is increased, the magnitude of the off-diagonal elements quickly decays, and hence all collective decay rates approach the single-atom value, $\gamma$.

In Fig. 2b we show the same collective decay rates for an atomic chain at $h=100 \mathrm{~nm}$ from a silica nanofiber with radius $r_{\mathrm{f}}=220 \mathrm{~nm}$. When the spacing between the atoms is much smaller than the transition wavelength, we observe that the collective decay rates do not change significantly from the ones in Fig. 2a. As $a / \lambda_{\mathrm{a}}$ is increased, however, a superradiant mode with enhanced decay rate splits from the rest. This superradiant mode corresponds to a guided rightward-propagating decay mode that emerges due to the presence of the fiber. This mode can also be observed in Fig. 2c, where the decay rates in the absence of free field are depicted. Here, we also identify a second (leftward-propagating) mode, which we will discuss later. Moreover, the subradiant decay modes present when considering coupling only to the nanofiber (Fig. 2c) lose their subradiant character in the hybrid situation (Fig. 2b). This sensitivity to the presence of unguided modes is particularly relevant for any works aiming at the creation of many-body subradiant states using emitters coupled to guided structures [21, 61, 62].

In order to gain an understanding of the nature of the superradiant mode, we show the magnitude and phase of its spatial profile $M_{S j}=\left|M_{S j}\right| \mathrm{e}^{i \varphi_{S j}}$ in Figs. $2 \mathrm{~d}$ and e for two values of $a / \lambda_{\mathrm{a}}$ and the three cases depicted in Figs. $2 \mathrm{a}, \mathrm{b}$ and c. For very small interatomic separation the spatial profile of all decay modes is independent of the presence of the fiber. As $a / \lambda_{\mathrm{a}}$ increases, the collective decay rates corresponding to the two most superradiant modes cross, and the profile of the "hybrid" superradiant mode becomes similar to the fully guided one (see black crosses and red plusses in panel d). For larger distances 


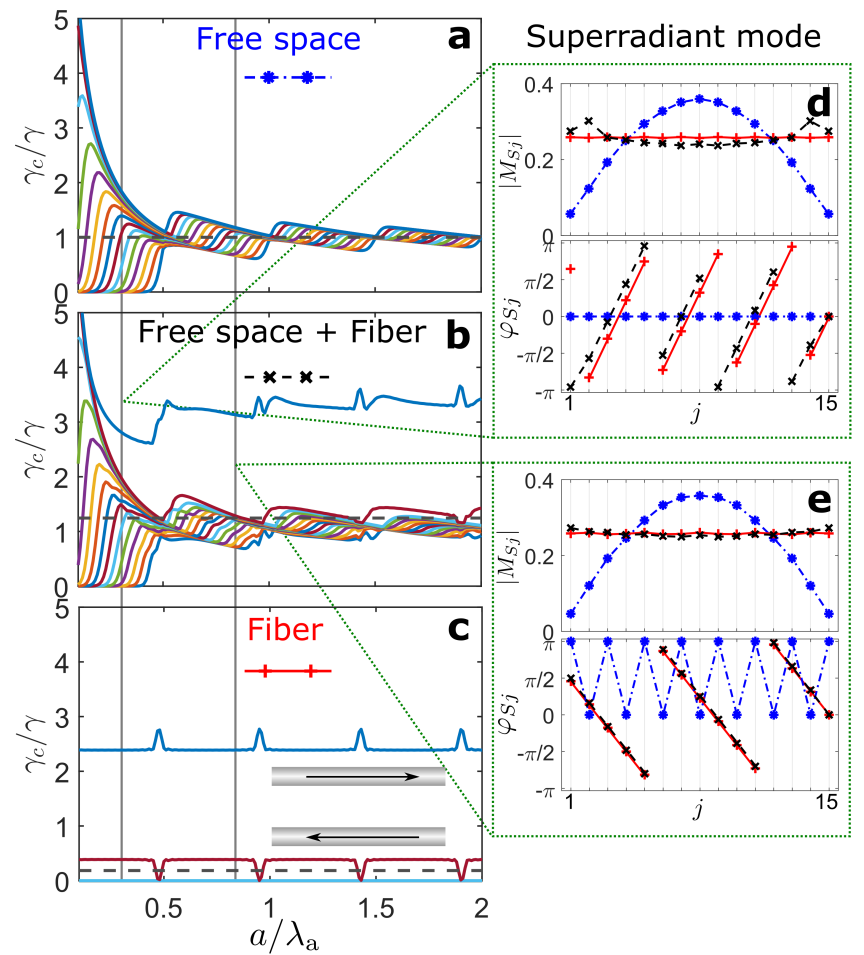

FIG. 2. Hybridization of decay modes. Collective decay rates $\gamma_{c}$ for a chain of $N=15$ atoms with transition wavelength $\lambda_{\mathrm{a}}=1 \mu \mathrm{m}$ a: in free space, b: considering both couplings to unguided free field modes and guided modes of a nanofiber at a distance $h=100 \mathrm{~nm}$ and c: only to the guided modes of the fiber (rightward and leftward-propagating modes indicated). The single atom decay rates are shown in each case for comparison (grey dashed lines). $\mathbf{d}$ and $\mathbf{e}$ : Magnitude $\left|M_{S i}\right|$ and phase $\varphi_{S i}$ of the superradiant decay mode's spatial profile for a chain with $a=250$ and $800 \mathrm{~nm}$, respectively, in free space (blue stars), coupled to the nanofiber guided modes (red plusses) and to both (black crosses).

between the atoms (panel e), this hybridized superradiant mode is almost completely formed by one of the fiber guided modes, as the almost identical mode profiles in Fig. 2e show.

Let us further analyze the phase profile of this superradiant mode, which will be key for understanding the collective enhancement of the rate and chirality of the guided photon emission. For the parameters chosen here, $72 \%$ of the photons that are emitted into the nanofiber from each single atom propagate rightwards, i.e., the single-atom guided coupling is chiral [65]. This symmetry breaking in the emission is manifested in the superradiant mode as a phase gradient across the chain. The phase difference between nearest neighbor atoms is $\Delta \phi=a \beta_{\mathrm{f}}$, with $\beta_{\mathrm{f}}$ being the propagation constant of the light inside the nanofiber (the value of $\beta_{\mathrm{f}}$ is close to $\left.2 \pi / \lambda_{\mathrm{a}}\right)$. Thus, every time that $a$ crosses a multiple of $\lambda_{\mathrm{a}} / 2$ an apparent change of sign of the phase gradient takes place (see, e.g. panels $\mathrm{d}$ and e in Fig. 2). In order to account for this aliasing, we rewrite the phase difference for nearest neighbors as $\Delta \phi=a \beta_{\mathrm{f}}-2 \pi n$, with $n=0,1, \ldots$ being the integer part of $a /\left(\lambda_{\mathrm{a}} / 2\right)$. Note as well that for the leftward-propagating mode (second highest decay rate state in Fig. 2c), the phase gradient has the opposite sign.

Collectively enhanced beta factor. The excitation of the superradiant mode which we just analyzed gives rise to an enhancement of both the rate and chirality of photon emission into the nanofiber. To investigate how this can be tested experimentally, we consider the response of the atomic chain when driven by a weak laser field (see scheme in Fig. 3). Its action is incorporated in the quantum master equation by making the substitution $\mathcal{H} \rightarrow \mathcal{H}+\sum_{i}\left[\Omega_{\mathrm{L}}\left(e^{i \mathbf{k}_{\mathrm{L}} \cdot \mathbf{r}_{i}} \sigma_{i}+e^{-i \mathbf{k}_{\mathrm{L}} \cdot \mathbf{r}_{i}} \sigma_{i}^{\dagger}\right)-\Delta \sigma_{i}^{\dagger} \sigma_{i}\right]$, where $\Omega_{\mathrm{L}}$ and $\Delta$ are the Rabi frequency and detuning of the laser from the $|g\rangle \rightarrow|e\rangle$ transition, respectively [66]. The laser imprints a phase pattern by tuning the angle $\varphi$ between its momentum $\mathrm{k}_{\mathrm{L}}$, and the chain. In order to match the phase profile of the rightward (superradiant), and leftward-propagating states that we introduced in the previous section, the laser angle must satisfy

$$
\cos \varphi= \pm\left(\frac{n \lambda_{\mathrm{a}}}{a}-\frac{\lambda_{\mathrm{a}}}{\lambda_{\mathrm{f}}}\right),
$$

with $\lambda_{\mathrm{f}}=2 \pi / \beta_{\mathrm{f}}$ and $n=1,2, \ldots$.

For the subsequent analysis, we define the total photon emission rate as $N_{p}(\Delta)=\sum_{i j} \Gamma_{i j}\left\langle\sigma_{i}^{\dagger} \sigma_{j}\right\rangle_{\mathrm{ss}}$, where $\langle\ldots\rangle_{\mathrm{ss}}$ denotes the expectation value in the stationary state [39]. This expectation value can be easily found in the limit of weak laser driving within the single-excitation subspace [65]. Analogously, the photon emission rate into the guided modes is given by $N_{p}^{\mathrm{g}}(\Delta)=\sum_{i j} \Gamma_{i j}^{\mathrm{g}}\left\langle\sigma_{i}^{\dagger} \sigma_{j}\right\rangle_{\mathrm{ss}}$, where the coefficients $\Gamma_{i j}^{\mathrm{g}}$ contain the couplings into the guided modes only. Moreover, we define the collective decay rate as the integral over the detuning of $N_{p}(\Delta)$. Similarly, we define the collective beta factor as the ratio between the total photon emission rate into the nanofiber (again integrated over $\Delta$ ) and the collective decay rate. Finally, the collective chirality is obtained by breaking down the emission rates into the right and left directions of the nanofiber. We define it as the difference between the guided photon emission rate into the rightward and leftward-propagating modes divided by the total photon emission rate into the nanofiber [65].

In Figs. 3a and b, we show $N_{p}$ and $N_{p}^{\mathrm{g}}$ for fixed values of the Rabi frequency of the laser $\Omega_{\mathrm{L}}$ and the ratio $a / \lambda_{\mathrm{a}}$ for a chain of $N=15$ atoms. The behavior strongly depends on the laser angle $\varphi$. For $\varphi=0$ (panel a), both the total and the guided photon rates have a characteristic Lorentzian shape only slightly shifted away from $\Delta=0$. Most importantly, the fraction of emission into the guided modes here is small for all values of the detuning. However, at $\varphi=1.37$ [solution of the equation (4) with $n=1$ ] this fraction is enhanced considerably, which 

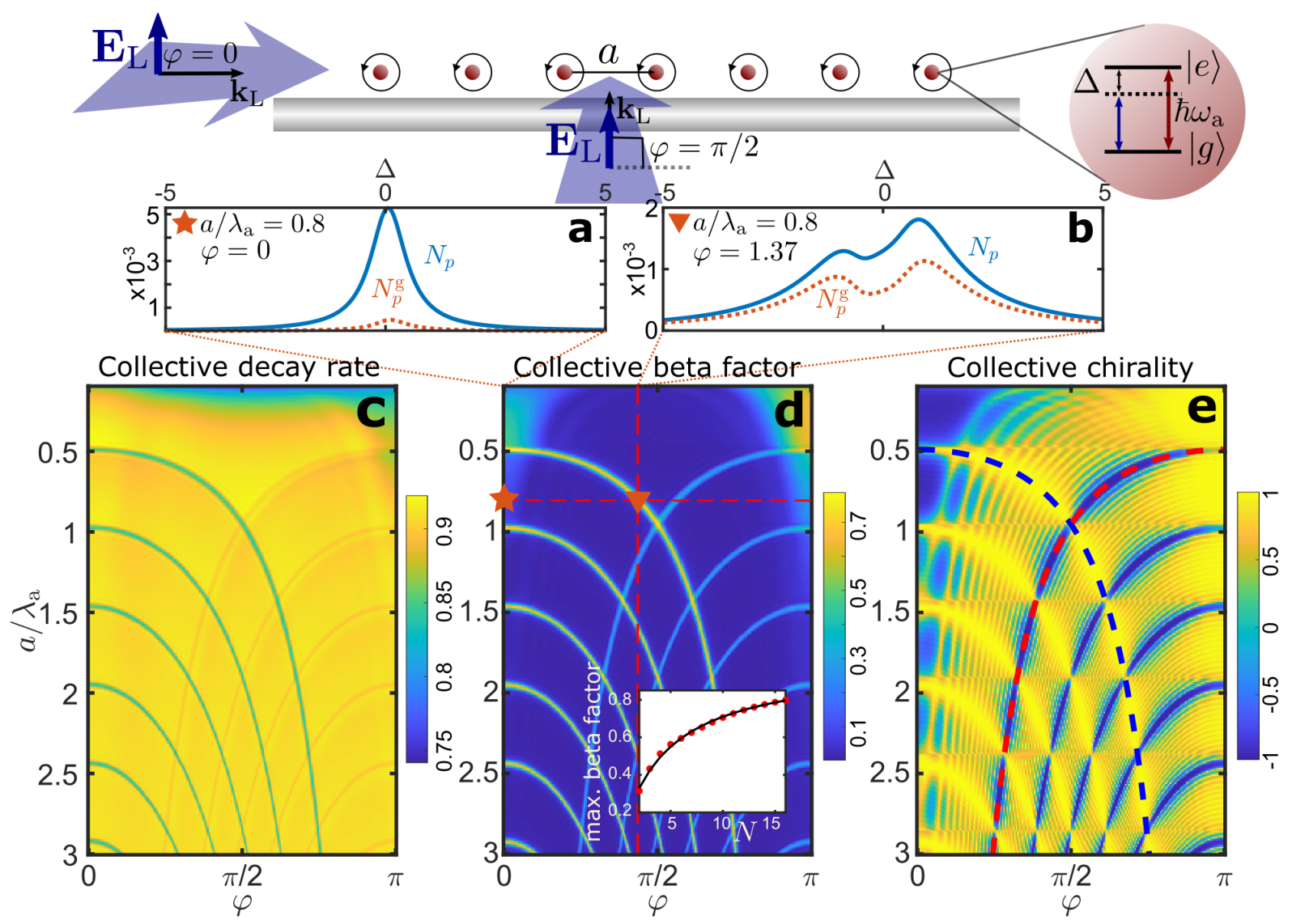

FIG. 3. Collectively enhanced emission properties. Scheme for the laser excitation of a chain of atoms located at $h=100 \mathrm{~nm}$ from a fiber with radius $r_{\mathrm{f}}=220 \mathrm{~nm}$. The Rabi frequency of the external laser field is $\Omega_{\mathrm{L}}=\gamma / 100$, and the angle between the k-vector of the laser and the orientation of the chain is $\varphi$. a and b: Total photon emission rate $N_{p}$ (blue solid line) and guided photon emission rate $N_{p}^{\mathrm{g}}$ (red dotted line) as a function of $\Delta$ (in units of the single atom decay rate $\gamma$ ) for $N=15$ at the two points shown in panel $\mathbf{d}$. c: Collective decay rate. d: Collective beta factor. Inset shows the maximum collective beta factor as a function of the system size $N$, red dots. The black line shows $N \Gamma^{\mathrm{g}} /\left(N \Gamma^{\mathrm{g}}+\Gamma^{\mathrm{u}}\right)$ (see text). e: Collective chirality. Shown for comparison is the expression (4) for $n=1$ (dashed lines).

is due to the angle of the laser momentum matching the phase profile of the superradiant state shown in Fig. 2e. Note, that while the superradiant mode is an eigenstate of the coefficient matrix $\Gamma_{i j}$, it is not an eigenstate of the effective Hamiltonian with coefficients $H_{i j}^{\text {eff }}=V_{i j}-i \Gamma_{i j} / 2$ that describes the dynamics of the system [65], which leads to the splitting of the superradiant peak into two, shifted away from resonance with different signs of the detuning.

In Figs. 3c-e we show the collective decay rate, beta factor, and chirality, as a function of $a / \lambda_{\mathrm{a}}$ and the laser angle $\varphi$. One clearly observes a collective modification of all quantities when the mode matching condition (4) is met, visible in a marked pattern of lines. First, the collective decay rate becomes smaller along these lines. Second, as predicted above, the collective beta factor is increased dramatically when the laser matches the most superradiant mode. This enhancement becomes more pronounced as the system size is increased, growing ap- proximately as $N \Gamma^{\mathrm{g}} /\left(N \Gamma^{\mathrm{g}}+\Gamma^{\mathrm{u}}\right)$ [39], with $\Gamma^{\mathrm{g} / \mathrm{u}}$ being the single atom emission rates into the guided and unguided modes [65]. This can be observed in the inset of Fig. 3d, where the maximum collective beta factor is shown as a function of $N$. Finally, the enhancement of the beta factor is accompanied by a dramatic increase of the chirality of the emission from its single-atom value $(0.72$ in the example shown here) to 0.999. I.e., the emission becomes nearly unidirectional [65]. Note that the laser angle can be chosen such that the second guided leftwardpropagating mode is excited (with a smaller beta factor), with chirality going up to -0.999 . Finally, we find that when $a$ is an integer multiple of $\lambda_{\mathrm{f}}$, the chirality recovers its single-atom value and the collective beta factor reaches its maximum at $\varphi=\pi / 2$. The reason is that here the phase profile of the two guided modes becomes flat $(\Delta \phi= \pm 2 \pi n)$ and, thus, the laser matches both modes simultaneously when its momentum is perpendicular to the chain. 
Conclusion and outlook. We show that the emission from a chain of atoms into the guided modes of a nanofiber can be collectively enhanced and made perfectly unidirectional. This can be achieved by mode matching the phase profile of an excitation laser to the phase gradient of a superradiant state emerging from the atom-fiber coupling. The parameters used in this work are achievable in current experimental setups [29-32].

A natural continuation to this work will be to investigate the properties and photon counting statistics (e.g. two-time correlations) of the light emitted into the nanofiber. Moreover, the challenge is to go beyond the weak excitation limit and to understand the fate of the collectively enhanced photon emission when the atoms are driven closer to saturation.

The authors acknowledge fruitful discussions with Philipp Schneeweiß and all members of the ErBeStA consortium. The research leading to these results has received funding from the European Union's H2020 research and innovation programme [Grant Agreement No. 800942 (ErBeStA)] and EPSRC [Grant No. EP/M014398/1]. IL acknowledges support from the "Wissenschaftler-Rückkehrprogramm GSO/CZS " of the Carl-Zeiss-Stiftung and the German Scholars Organization e.V.. BO was supported by the Royal Society and EPSRC [Grant No. DH130145]. BL is supported by the Leverhulme Trust through the research project Grant No. RPG-2018-213.

[1] R. H. Dicke, "Coherence in spontaneous radiation processes," Phys. Rev., vol. 93, p. 99, 1954.

[2] G. S. Agarwal, "Master-equation approach to spontaneous emission," Phys. Rev. A, vol. 2, p. 2038, 1970.

[3] R. H. Lehmberg, "Radiation from an $n$-atom system. I. general formalism," Phys. Rev. A, vol. 2, p. 883, 1970.

[4] J. Keaveney, A. Sargsyan, U. Krohn, I. G. Hughes, D. Sarkisyan, and C. S. Adams, "Cooperative lamb shift in an atomic vapor layer of nanometer thickness," Phys. Rev. Lett., vol. 108, p. 173601, 2012.

[5] J. Pellegrino, R. Bourgain, S. Jennewein, Y. R. P. Sortais, A. Browaeys, S. D. Jenkins, and J. Ruostekoski, "Observation of suppression of light scattering induced by dipole-dipole interactions in a cold-atom ensemble," Phys. Rev. Lett., vol. 113, p. 133602, 2014.

[6] R. J. Bettles, S. A. Gardiner, and C. S. Adams, "Cooperative eigenmodes and scattering in one-dimensional atomic arrays," Phys. Rev. A, vol. 94, p. 043844, 2016.

[7] R. T. Sutherland and F. Robicheaux, "Collective dipoledipole interactions in an atomic array," Phys. Rev. A, vol. 94, p. 013847, 2016.

[8] M. O. Araújo, I. Krešić, R. Kaiser, and W. Guerin, "Superradiance in a large and dilute cloud of cold atoms in the linear-optics regime," Phys. Rev. Lett., vol. 117, p. 073002, 2016.

[9] W. Guerin, M. O. Araújo, and R. Kaiser, "Subradiance in a large cloud of cold atoms," Phys. Rev. Lett., vol. 116, p. $083601,2016$.

[10] H. T. Dung, L. Knöll, and D.-G. Welsch, "Resonant dipole-dipole interaction in the presence of dispersing and absorbing surroundings," Phys. Rev. A, vol. 66, p. 063810, 2002.

[11] M. Wallquist, K. Hammerer, P. Rabl, M. Lukin, and P. Zoller, "Hybrid quantum devices and quantum engineering," Physica Scripta, vol. T137, p. 014001, 2009.

[12] F. Zhou, Y. Liu, and Z.-Y. Li, "Surface-plasmonpolariton-assisted dipole-dipole interaction near metal surfaces," Opt. Lett., vol. 36, p. 1969, 2011.

[13] D. Martín-Cano, A. González-Tudela, L. Martín-Moreno, F. J. García-Vidal, C. Tejedor, and E. Moreno, "Dissipation-driven generation of two-qubit entanglement mediated by plasmonic waveguides," Phys. Rev. B, vol. 84, p. 235306, 2011.

[14] C. Stehle, C. Zimmermann, and S. Slama, "Cooperative coupling of ultracold atoms and surface plasmons," Nat. Phys., vol. 10, pp. 937-942, 2014.

[15] J. Hou, K. Słowik, F. Lederer, and C. Rockstuhl, "Dissipation-driven entanglement between qubits mediated by plasmonic nanoantennas," Phys. Rev. B, vol. 89, p. 235413, 2014.

[16] Y. Chao, J. Sheng, J. A. Sedlacek, and J. P. Shaffer, "Surface phonon polaritons on anisotropic piezoelectric superlattices," Phys. Rev. B, vol. 93, p. 045419, 2016.

[17] R. Jones, J. A. Needham, I. Lesanovsky, F. Intravaia, and B. Olmos, "Modified dipole-dipole interaction and dissipation in an atomic ensemble near surfaces," Phys. Rev. A, vol. 97, p. 053841, 2018.

[18] E. M. Purcell, "Spontaneous emission probabilities at radio frequencies," Phys. Rev., vol. 69, p. 681, 1946.

[19] R. Spreeuw, V. Ivanov, R. A. Cornelussen, and H. B. van Linden van den Heuvell, "Qed-modified radiative properties and dynamics of cold atoms moving through an evanescent wave," Optics and Spectroscopy, vol. 99, pp. 459-465, 012005.

[20] E. Fort and S. Grésillon, "Surface enhanced fluorescence," J. Phys. D: App. Phys., vol. 41, p. 013001, 2008.

[21] A. Asenjo-Garcia, M. Moreno-Cardoner, A. Albrecht, H. J. Kimble, and D. E. Chang, "Exponential improvement in photon storage fidelities using subradiance and "selective radiance" in atomic arrays," Phys. Rev. X, vol. 7, p. 031024, 2017.

[22] A. F. Kockum, G. Johansson, and F. Nori, "Decoherencefree interaction between giant atoms in waveguide quantum electrodynamics," Phys. Rev. Lett., vol. 120, p. 140404, 2018.

[23] A. Yariv, Optical Electronics in Modern Communications, ch. 3. Oxford University Press, 1997.

[24] V. I. Balykin, K. Hakuta, F. Le Kien, J. Q. Liang, and M. Morinaga, "Atom trapping and guiding with a subwavelength-diameter optical fiber," Phys. Rev. A, vol. 70, p. 011401, 2004.

[25] F. Le Kien, J. Q. Liang, K. Hakuta, and V. I. Balykin, "Field intensity distributions and polarization orientations in a vacuum-clad subwavelength-diameter optical fiber," Opt. Commun, vol. 242, p. 445, 2004.

[26] Y. Chen, T. R. Nielsen, N. Gregersen, P. Lodahl, and J. Mørk, "Finite-element modeling of spontaneous emission of a quantum emitter at nanoscale proximity to plasmonic waveguides," Phys. Rev. B, vol. 81, p. 125431, 2010.

[27] K. Y. Bliokh, F. J. Rodríguez-Fortuño, F. Nori, and A. V. 
Zayats, "Spin-orbit interactions of light," Nat. Photonics, vol. 9, p. 796, 2015.

[28] Note that we use here the terms chiral and chirality to describe directionality, i.e., the breaking of the forwardsbackwards propagation in these systems.

[29] R. Mitsch, C. Sayrin, B. Albrecht, P. Schneeweiss, and A. Rauschenbeutel, "Quantum state-controlled directional spontaneous emission of photons into a nanophotonic waveguide," Nat. Commun, vol. 5, p. 5713, 2014.

[30] M. Scheucher, A. Hilico, E. Will, J. Volz, and A. Rauschenbeutel, "Quantum optical circulator controlled by a single chirally coupled atom," Science, vol. 354, no. 6319, pp. 1577-1580, 2016.

[31] P. Solano, P. Barberis-Blostein, F. K. Fatemi, L. A. Orozco, and L. Rolston, "Super-radiance reveals infiniterange dipole interactions through a nanofiber," Nat. Commun, vol. 8, p. 1857, 2017.

[32] A. Dareau, Y. Meng, P. Schneeweiss, and A. Rauschenbeutel, "Observation of ultrastrong spin-motion coupling for cold atoms in optical microtraps," Phys. Rev. Lett., vol. 121, p. 253603, 2018.

[33] P. Mrowiński, P. Schnauber, P. Gutsche, A. K. J. Schall, S. Burger, S. Rodt, and S. Reitzenstein, "Directional emission of a deterministically fabricated quantum dot-bragg reflection multimode waveguide system," $A C S$ Photonics, vol. 6, pp. 2231-2237, 2019.

[34] L. Scarpelli, B. Lang, F. Masia, D. M. Beggs, E. A. Muljarov, A. B. Young, R. Oulton, M. Kamp, S. Höfling, C. Schneider, and W. Langbein, "99\% beta factor and directional coupling of quantum dots to fast light in photonic crystal waveguides determined by spectral imaging," Phys. Rev. B, vol. 100, p. 035311, 2019.

[35] V. V. Klimov and M. Ducloy, "Spontaneous emission rate of an excited atom placed near a nanofiber," Phys. Rev. A, vol. 69, p. 013812, 2004.

[36] F. Le Kien, S. Dutta Gupta, V. I. Balykin, and K. Hakuta, "Spontaneous emission of a cesium atom near a nanofiber: Efficient coupling of light to guided modes," Phys. Rev. A, vol. 72, p. 032509, 2005.

[37] F. Le Kien and A. Rauschenbeutel, "Anisotropy in scattering of light from an atom into the guided modes of a nanofiber," Phys. Rev. A, vol. 90, p. 023805, 2014.

[38] R. J. Coles, D. M. Price, J. E. Dixon, B. Royall, E. Clarke, P. Kok, M. S. Skolnick, A. M. Fox, and M. N. Makhonin, "Chirality of nanophotonic waveguide with embedded quantum emitter for unidirectional spin transfer," Nat. Comm., vol. 7, p. 11183, 2016.

[39] F. L. Kien and K. Hakuta, "Cooperative enhancement of channeling of emission from atoms into a nanofiber," Phys. Rev. A, vol. 77, p. 013801, 2008.

[40] D. Dzsotjan, A. S. Sørensen, and M. Fleischhauer, "Quantum emitters coupled to surface plasmons of a nanowire: A green's function approach," Phys. Rev. B, vol. 82, p. 075427, 2010.

[41] D. Dzsotjan, J. Kästel, and M. Fleischhauer, "Dipoledipole shift of quantum emitters coupled to surface plasmons of a nanowire," Phys. Rev. B, vol. 84, p. 075419, 2011.

[42] Y.-L. L. Fang, H. Zheng, and H. U. Baranger, "Onedimensional waveguide coupled to multiple qubits: photon-photon correlations," EPJ Quantum Technol., vol. 1, p. 3, 2014.

[43] Y.-L. L. Fang and H. U. Baranger, "Waveguide qed: Power spectra and correlations of two photons scattered off multiple distant qubits and a mirror," Phys. Rev. A, vol. 91, p. 053845, 2015.

[44] C. Gonzalez-Ballestero, A. Gonzalez-Tudela, F. J. Garcia-Vidal, and E. Moreno, "Chiral route to spontaneous entanglement generation," Phys. Rev. B, vol. 92, p. 155304, 2015.

[45] D. F. Kornovan, A. S. Sheremet, and M. I. Petrov, "Collective polaritonic modes in an array of two-level quantum emitters coupled to an optical nanofiber," Phys. Rev. $B$, vol. 94, p. 245416, 2016.

[46] F. Le Kien and A. Rauschenbeutel, "Nanofiber-mediated chiral radiative coupling between two atoms," Phys. Rev. A, vol. 95, p. 023838, 2017.

[47] J. Ruostekoski and J. Javanainen, "Emergence of correlated optics in one-dimensional waveguides for classical and quantum atomic gases," Phys. Rev. Lett., vol. 117, p. $143602,2016$.

[48] J. Ruostekoski and J. Javanainen, "Arrays of strongly coupled atoms in a one-dimensional waveguide," Phys. Rev. A, vol. 96, p. 033857, 2017.

[49] I. M. Mirza and J. C. Schotland, "Multiqubit entanglement in bidirectional-chiral-waveguide qed," Phys. Rev. A, vol. 94, p. 012302, 2016.

[50] I. M. Mirza and J. C. Schotland, "Two-photon entanglement in multiqubit bidirectional-waveguide qed," Phys. Rev. A, vol. 94, p. 012309, 2016.

[51] I. M. Mirza, J. G. Hoskins, and J. C. Schotland, "Chirality, band structure, and localization in waveguide quantum electrodynamics," Phys. Rev. A, vol. 96, p. 053804, 2017.

[52] M.-T. Cheng, J. Xu, and G. S. Agarwal, "Waveguide transport mediated by strong coupling with atoms," Phys. Rev. A, vol. 95, p. 053807, 2017.

[53] K. Stannigel, P. Rabl, A. S. Sørensen, M. D. Lukin, and P. Zoller, "Optomechanical transducers for quantuminformation processing," Phys. Rev. A, vol. 84, p. 042341, 2011.

[54] K. Stannigel, P. Rabl, and P. Zoller, "Driven-dissipative preparation of entangled states in cascaded quantumoptical networks," New J. Phys., vol. 14, no. 6, p. 063014, 2012.

[55] I. Söllner, S. Mahmoodian, S. L. Hansen, L. Midolo, A. Javadi, G. Kiršanskè, T. Pregnolato, H. El-Ella, E. H. Lee, J. D. Song, S. Stobbe, and P. Lodahl, "Deterministic photon-emitter coupling in chiral photonic circuits," Nat. Nanotechnol., vol. 10, p. 775, 2015.

[56] T. Ramos, H. Pichler, A. J. Daley, and P. Zoller, "Quantum spin dimers from chiral dissipation in cold-atom chains," Phys. Rev. Lett., vol. 113, p. 237203, 2014.

[57] H. Pichler, T. Ramos, A. J. Daley, and P. Zoller, "Quantum optics of chiral spin networks," Phys. Rev. A, vol. 91, p. 042116, 2015.

[58] S. Mahmoodian, P. Lodahl, and A. S. Sørensen, "Quantum networks with chiral-light-matter interaction in waveguides," Phys. Rev. Lett., vol. 117, p. 240501, 2016.

[59] B. Vermersch, P.-O. Guimond, H. Pichler, and P. Zoller, "Quantum state transfer via noisy photonic and phononic waveguides," Phys. Rev. Lett., vol. 118, p. 133601, 2017.

[60] H. H. Jen, "Selective transport of atomic excitations in a driven chiral-coupled atomic chain," J. Phys. B: At. Mol. Opt. Phys., vol. 52, no. 6, p. 065502, 2019.

[61] G. Buonaiuto, R. Jones, B. Olmos, and I. Lesanovsky, "Dynamical creation and detection of entangled manybody states in a chiral atom chain," New J. Phys., vol. 21, 
p. 113021, 2019.

[62] S. Mahmoodian, G. Calajó, D. E. Chang, K. Hammerer, and A. S. Sørensen, "Dynamical creation and detection of entangled many-body states in a chiral atom chain," arXiv:1910.05828, 2019.

[63] A. W. Snyder and J. D. Love, Optical Waveguide Theory, ch. 11. Chapman and Hall, 1983.

[64] I. H. Malitson, "Interspecimen comparison of the refractive index of fused silica," J. Opt. Soc. Am., vol. 55, p. $1205,1965$.

[65] See Supplemental Material at URL for technical details on the Master equation coeffcients, the single-atom emis- sion properties, dynamics in the single-excitation subspace, definitions of collective emission properties and simulations of the emission dynamics from classical radiating dipoles.

[66] Note that here for the two-level approximation to be valid in a multilevel atom, one can consider the presence of a uniform magnetic field in the $y$-direction that splits the levels, making the laser resonant only with the desired transition. Hence, the detuning of the laser must be smaller than this energy level shift. 